\title{
SIMPLE SAMPLING DEVICE FOR CAPILLARY ISOTACHOPHORESIS AND CAPILLARY ZONE ELECTROPHORESIS
}

Th. P. E. M. VERHEGGEN, J. L. BECKERS and F. M. EVERAERTS*

Laboratory of Instrumental Analysis, Eindhoven University of Technology, P.O. Box 513, 5600 MB

Eindhoven (The Netherlands)

SUMMARY

A simple sampling device for isotachophoresis and zone electrophoresis is described, whereby the sample solution is introduced directly into a broadened part of the capillary tube by two feeders, placed perpendicularly to the capillary tube. The advantage of this sampling device is that with the absence of moving parts, automation of the sample introduction can be carried out in a simple way, cleaning is simple and the device is inexpensive. Experiments showed that a reproducibility of less than $2 \%$ can be obtained, including the day to day variations using different sample solutions.

\section{INTRODUCTION}

The sampling methods usually applied in capillary isotachophoresis (ITP) and capillary zone electrophoresis (CZE) are: injection with a syringe, via a septum, in an injection block, injection valves, with/without a sample loop, and dipping one end of the capillary tube into the sample solution, whereby the sample is introduced by (a) the gravity flow or (b) electroendosmosis and/or electromigration.

The best way of sampling in CZE is to introduce a representative aliquot of a sample, concentrated as much as possible in a narrow band and without mixing with the background electrolyte ${ }^{1}$. However, none of the methods mentioned above was appropriate for this purpose. The use of a syringe can cause reading errors, especially for small amounts of sample, and a great disadvantage is the mixing of the sample with the background ionic species so that the sample cannot be introduced in a narrow band. In ITP this is of no importance because of the self-correcting effect of the zone boundaries.

A sample valve is ${ }^{1}$, in fact, the most suitable sampling method for CZE. With a sample valve a known volume of a representative aliquot of the sample can be introduced without mixing with the background electrolyte. Using a sample valve in CZE it was very difficult, however, to avoid impurities coming from the "dead volumes" (i.e., impurities coming from the liquid films) between moving parts because in CZE very small amounts of ionic species can be detected.

When a gravity flow ${ }^{2}$, electroendosmosis or electromigration ${ }^{3,4}$ is applied an 
unknown volume is introduced so that an internal standard has to be used for quantitative analyses. In electroendosmosis and electromigration, moreover, the sample introduced often does not have the same composition as the original sample (see Discussion). Applying pulse injections with splitting techniques ${ }^{5}$ can be considered as electromigration with the advantage that a representative aliquot of the sample is taken.

In this paper a simple sampling device for capillary ITP and CZE is described, whereby the sample solution is introduced directly into part of the capillary tube by means of two feeders, perpendicular to the capillary tube. The sample can be introduced without mixing with the background electrolyte. With the absence of moving parts cleaning is simple. An advantage, as obtained with the use of a valve, is the concentration effect of dilute sample ionic species ${ }^{6}$.

\section{EXPERIMENTAL}

In order to examine the utility of this new sampling device, an apparatus was constructed as shown in Fig. 1. A cast capillary block $\mathrm{C}$ is connected between the electrode compartment $A_{1}$ and the sampling device SD. In the electrode compartments $A_{1}$ and $A_{2}$, the electrolyte solutions contact the Pt-Ir electrodes E. The capillary tube $(0.25 \mathrm{~mm}$ I.D., separation length $6 \mathrm{~cm})$ contains measuring electrodes $M$ connected with a.c. electronics 7 . The sampling device SD consists of a broadened part of the capillary tube $(0.55 \mathrm{~mm}$ I.D. $)$ connected with two feeders $(0.4 \mathrm{~mm}$ diameter), perpendicular to the capillary tube.

The electrolyte solutions are introduced using valves 1 and 3 and the drain 2 . Valves 5 and 6 are used for complete rinsing of the electrode compartments. The sample can be introduced via valve 4 and the drain 2 .

In first instance, the experiments were carried out with a closed valve, connected to drain 2. All data presented in this paper, however, were measured with drain 2 open, because identical results were obtained.

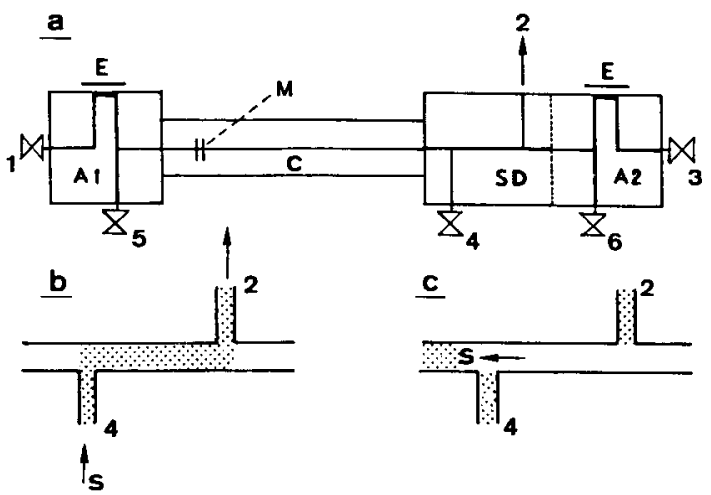

Fig. 1. (a) Schematic diagram of electrophoretic equipment with sample device (for further explanation, see text). (b) Sample device during sampling ( $\mathrm{S}=$ sample). (c) Sample device after a certain time ( $\mathrm{S}=$ sample). 


\section{RESULTS}

In order to study the feasibility, accuracy and reproducibility of the new sampling method, two questions have to be answered. The first is whether the sample device (SD) has a constant volume independent of the mobilities and concentrations of the sample components and the exact value of this SD volume. It is possible that, although no electric field gradient over the feeders exists, sample components from the feeders may diffuse into the capillary tube when the sample has already left the sampling position. This means that these sample ions, in the ITP mode, will be found in the terminating zone (with a very high electric field gradient) by means of which some of the sample ionic species can reach their own sample zones. This effect will lead to a greater zone length for high ionic mobilities and high concentrations.

The second question is whether reproducible and linear calibration graphs can be obtained for each sample component in both the ITP and CZE modes.

\section{Determination of the volume of the sample device}

For the determination of the SD volume, the fact has been used that equal amounts of ionic species always need equal times to pass a detector, whatever capillary diameters may be used in ITP analyses, provided that an equal electric current is applied.

Because in ITP the equation

$$
E=j \sigma
$$

holds, where $j$ is the electric current density in $\mathrm{A} / \mathrm{cm}^{2}, \sigma$ is the specific resistance in $\Omega \mathrm{cm}$ and $E$ is the electric field gradient in $\mathrm{V} / \mathrm{cm}$, the concentration of a specific sample zone is always constant if the same leading electrolyte is used. If, for example, the cross-sectional area of the capillary tube is increased, then the zone length decreases in proportion. Because the electric current density $j$ decreases in a similar way, the zone velocity decreases, hence the time needed for the zone to pass the detector remains the same. This can also be formulated in another way, as follows. In order that a specific amount of a sample can pass a detector, a specific quantity of charge $Q$ has to be transported. The quantity of transported charge will always be:

$$
Q=I t
$$

Working at a constant electric current $I$ a specific quantity of charge $Q$ will pass in an equal time $t$. This fact was used to determine the volume of the sampling device.

Several different volumes of a sample solution were introduced into an ITP apparatus equipped with a capillary tube of $0.2 \mathrm{~mm}$ I.D. and an injection block. The sample volumes were introduced with a syringe and the volumes were read off under a microscope. Using the corresponding zone lengths a calibration graph was constructed.

The same sample solution was then introduced by the SD into the apparatus described in Fig. 1, and an ITP experiment was carried out using the same leading electrolyte and the same electric current. From the measured zone length the SD 


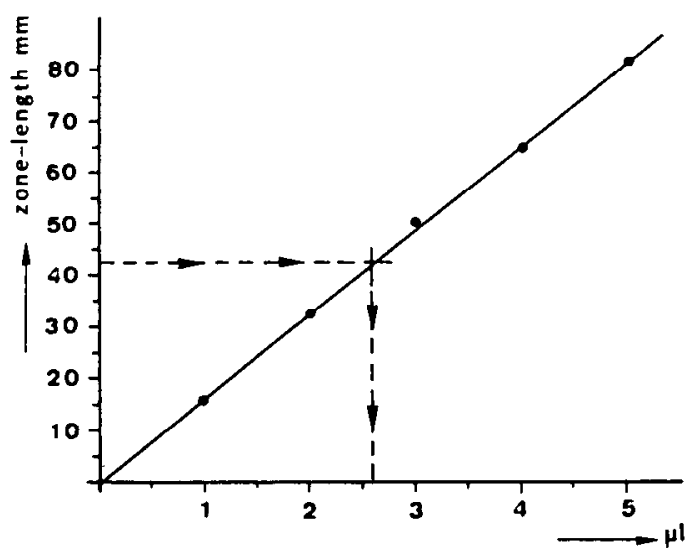

Fig. 2. Calibration graph for glutamate $(0.001 M)$ in a leading electrolyte system at pH $6(0.01 M$ hydrochloric acid + and $0.02 M$ histidine). The sample was introduced with a syringe and an injection block. From the zone length obtained using the sample device (SD) its volume can be obtained (dotted line).

volume can be obtained using the calibration graph for the ITP apparatus with injection block (see Fig. 2). This was carried out for different electrolyte systems and different sample solutions and the values obtained are given in Table I. It can be concluded from Table I that for ionic species with high ionic mobilities, a larger sample volume is found using the SD.

In order to establish whether the SD volume is also dependent on the concentration of the sample ionic species, the experiments were repeated with both apparatus for glutamate at different concentrations. The results are given in Table II, from which it can be concluded that the SD volume also depends on the concentration of the sample ionic species.

\section{TABLE I}

ZONE LENGTHS MEASURED WITH ITP EQUIPMENT WITH AN INJECTION BLOCK AND WITH THE SD FOR IONIC SPECIES AT A CONCENTRATION OF $0.001 M$, AND CALCULATED SD VOLUMES

The leading electrolytes were $0.01 M$ hydrochloric acid $+0.02 M$ histidine at $\mathrm{pH} 6$ and $0.01 M$ hydrochloric acid $+0.02 M \varepsilon$-aminocaproic acid at $\mathrm{pH}$ 4.4. The terminator was MES. From the calibration graphs obtained with the injection block data and the zone length of the SD, the SD volume was calculated. The electric current was $25 \mu \mathrm{A}$.

\begin{tabular}{|c|c|c|c|c|c|c|c|c|}
\hline \multirow[t]{2}{*}{ Ion } & \multirow[t]{2}{*}{$p H$} & \multicolumn{5}{|c|}{ Zone length using injection block ( $\mathrm{mm}$ ) } & \multirow{2}{*}{$\begin{array}{l}\text { Zone length } \\
\text { using } S D \\
(\mathrm{~mm})\end{array}$} & \multirow{2}{*}{$\begin{array}{l}S D \\
\text { volume } \\
(\mu l)\end{array}$} \\
\hline & & $I \mu l^{\star}$ & $2 \mu l^{\star}$ & $3 \mu l^{\star}$ & $4 \mu l^{\star}$ & $5 \mu l^{\star}$ & & \\
\hline Nitrate & 6 & 13.7 & 24.4 & 36.7 & 48.3 & 59.1 & 34.1 & 2.80 \\
\hline Propionate & & 13.0 & 26.1 & 38.9 & 52.2 & 64.3 & 35.2 & 2.71 \\
\hline Glutamate & & 15.3 & 32.4 & 48.5 & 65.5 & 80.6 & 42.0 & 2.60 \\
\hline Nitrate & 4.4 & 13.5 & 24.9 & 36.0 & 49.1 & 60.4 & 34.3 & 2.82 \\
\hline Propionate & & 12.7 & 25.6 & 37.4 & 52.0 & 64,3 & 33.5 & 2.61 \\
\hline Glutamate & & 14.8 & 31.6 & 46.7 & 62.7 & 77.3 & 39.5 & 2.54 \\
\hline
\end{tabular}

* Injection volume. 
TABLE II

AS TABLE I FOR GLUTAMATE SOLUTIONS OF DIFFERENT CONCENTRATIONS

\begin{tabular}{|c|c|c|c|c|c|c|c|}
\hline \multirow{2}{*}{$\begin{array}{l}\text { Concentration } \\
\text { (M) }\end{array}$} & \multicolumn{5}{|c|}{ Zone length using injection block ( $\mathrm{mm}$ ) } & \multirow{2}{*}{$\begin{array}{l}\text { Zone length } \\
\text { using SD } \\
(\mathrm{mm})\end{array}$} & \multirow{2}{*}{$\begin{array}{l}\text { SD volume } \\
(\mu l)\end{array}$} \\
\hline & $I \mu I^{*}$ & $2 \mu l^{\star}$ & $3 \mu l^{\star}$ & $4 \mu l^{\star}$ & $5 \mu l^{\star}$ & & \\
\hline 0.005 & 79.5 & 162.5 & 244.3 & 323.0 & 416.3 & 206.1 & 2.54 \\
\hline 0.001 & 16.1 & 32.6 & 50.1 & 64.8 & 81.4 & 42.5 & 2.60 \\
\hline 0.0005 & 8.1 & 16.1 & 24.4 & 33.5 & 40.2 & 21.6 & 2.65 \\
\hline
\end{tabular}

* Injection volume.

The diffusion of sample ionic species from the feeders into the capillary tube cannot explain these two effects. The results in Table I, viz., larger effective SD volumes for ionic species with high mobilities, agree with the possibility of diffusion from the feeders, but the results in Table II, viz., higher effective SD volumes for smaller sample concentrations, do not. The explanation for the latter may possibly be that introducing low concentrations of sample ionic species means that the terminator solution at the original position of sampling will also have a low concentration according to Kohlrausch's law ${ }^{8}$. Hence high electric field strengths and high temperatures will result, which may be responsible for the larger effective SD volumes. The differences in SD volumes are, however, only a few percent.

\section{Calibration}

In order to check the reproducibility and linearity of the SD we measured the zone lengths for glutamate and acetate in an electrolyte system at $\mathrm{pH} 6$ for several concentrations. The different concentrations were prepared by dilution of two different prepared standard solutions. The results are given in Table III, from which it can be concluded that linear calibration graphs were obtained with good reproducibility for the ionic species (see Table IV).

In order to check whether the calibration graphs are affected by the presence of other ionic species in the sample, we prepared several mixtures of acetate and glutamate and used the measured zone lengths to determine their concentrations in the sample mixture using the calibration graphs. In all instances the differences were less than about $2 \%$.

To compare the results obtained with an injection block and the new SD, in Table IV all data for the calibration graphs are given. The results of a regression analysis are given, viz., the regression coefficient and the constants $a$ and $b$ according to the equation

$$
Y=a X+b
$$

where $Y$ is the zone length $(\mathrm{mm})$ and $X$ is the volume injected $(\mu l)$ or the concentration $(M)$.

From Table IV it can be concluded that the regression coefficients for the new sample device are better than those for the injection block. Although we used the average value of the zone length from five experiments for the new $\mathrm{SD}$, it must be 


\section{TABLE III}

\section{ZONE LENGTHS FOR SEVERAL CONCENTRATIONS OF ACETATE AND GLUTAMATE MEASURED USING THE SD}

All zone lengths were measured five times on different days. The leading electrolyte was $0.01 M$ hydrochloric acid $+0.02 M$ histidine at $\mathrm{pH}$ 6. The terminator was MES. The electric current was $25 \mu \mathrm{A}$.

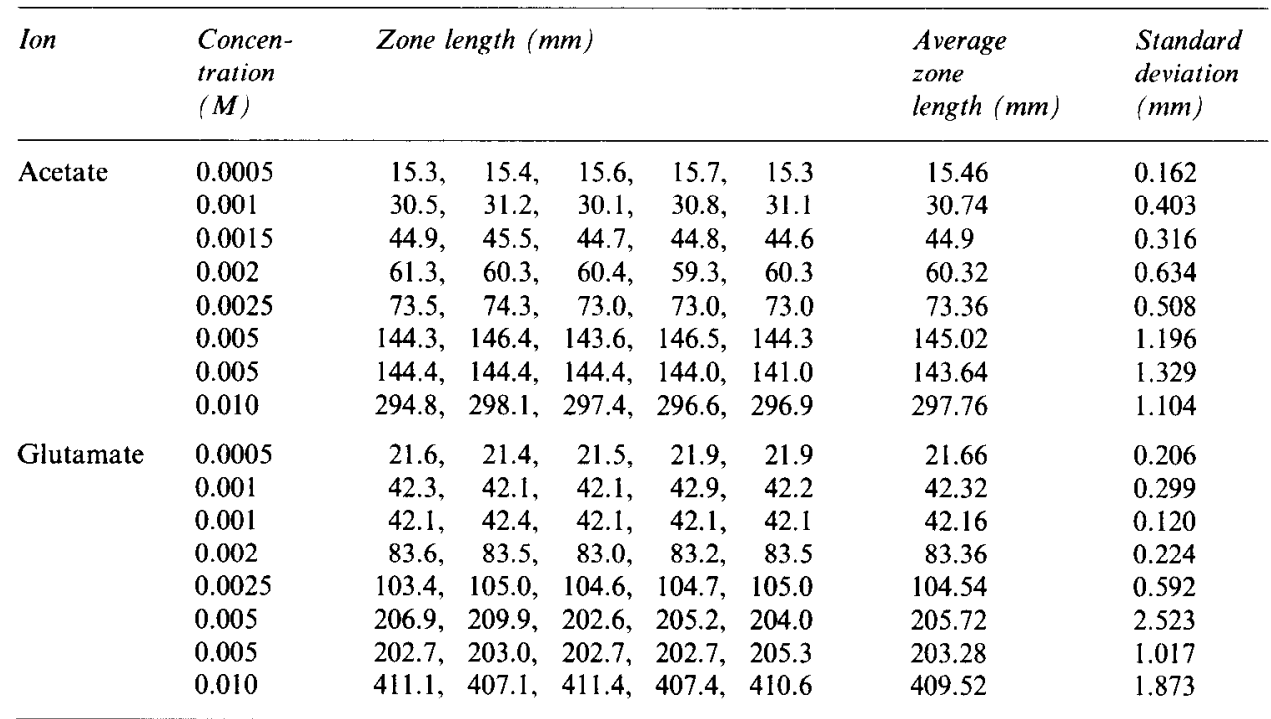

\section{TABLE IV}

CORRELATION COEFFICIENT AND THE CONSTANTS $a$ AND $b$ FOR THE REGRESSION ANALYSIS OF ALL CALIBRATION GRAPHS

\begin{tabular}{|c|c|c|c|c|c|}
\hline Apparatus & $p H$ & Ion & $\begin{array}{l}\text { Constant a } \\
(\mathrm{mm} / \mu \mathrm{l})\end{array}$ & $\begin{array}{l}\text { Constant } b \\
(\mathrm{~mm})\end{array}$ & $\begin{array}{l}\text { Correlation } \\
\text { coefficient }\end{array}$ \\
\hline \multirow[t]{6}{*}{ Injection block ${ }^{\star}$} & \multirow[t]{3}{*}{6} & Nitrate & 11.47 & 2.03 & 0.99950 \\
\hline & & Propionate & 12.87 & 0.29 & 0.99980 \\
\hline & & Glutamate & 16.19 & 0.0 & 0.99945 \\
\hline & \multirow[t]{3}{*}{4.4} & Nitrate & 12.18 & 0.0 & 0.99805 \\
\hline & & Propionate & 12.83 & 0.0 & 0.99899 \\
\hline & & Glutamate & 15.55 & 0.0 & 0.99948 \\
\hline \multirow[t]{3}{*}{ Injection block ${ }^{\star \star}$} & \multirow[t]{3}{*}{6} & Glutamate $(0.005 M)$ & 83.41 & -5.11 & 0.99925 \\
\hline & & Glutamate $(0.001 M)$ & 16.28 & 0.16 & 0.99940 \\
\hline & & Glutamate $(0.0005 M)$ & 8.16 & -0.02 & 0.99827 \\
\hline \multirow[t]{2}{*}{$\mathrm{SD}^{\star \star \star}$} & \multirow[t]{2}{*}{6} & Acetate & $29409.6^{\S}$ & 0.18 & 0.99954 \\
\hline & & Glutamate & $40752.2^{\S}$ & 1.53 & 0.99995 \\
\hline
\end{tabular}

* Results in Table I.

* Results in Table II.

$\star \star \star$ Results in Table III.

$\$ \mathrm{~mm} \cdot \mathrm{mol}^{-1} \cdot 1$. 
borne in mind that all these experiments were carried out with different electrolyte solutions and on different days so that these deviations include all possible errors such as those coming from the sample device, day to day variations, the use of different stock solutions and the non-linearity of the calibration graphs.

From the foregoing experiments it can be concluded that the new SD has an effective volume that is dependent on the effective mobilities and concentrations of the sample ionic species. Calibration graphs, for the different ionic species, however, show a linear relationship between zone length and concentration of ionic species, independent on their mobilities and unaffected by the presence of other sample ionic species.

The dimensions of the feeders and the sampling channel were chosen arbitrarily. The choice of the optimal dimensions of the SD is under investigation, especially for the use of narrow-bore capillaries $(50 \mu \mathrm{m})$.

In order to study the possibilities of automation, some experiments were carried out in which all electrolyte solutions and the sample were introduced by aspiration, using a simple peristaltic pump connected to drain 2 . The results were identical with those obtained in the manual experiments, which means that the SD can be used for the automation of analyses.

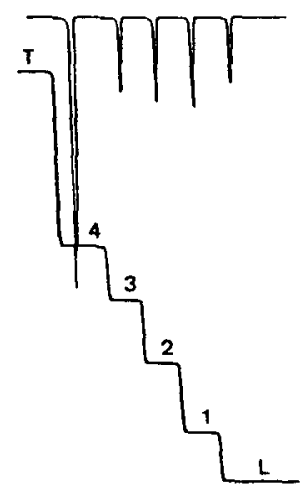

a
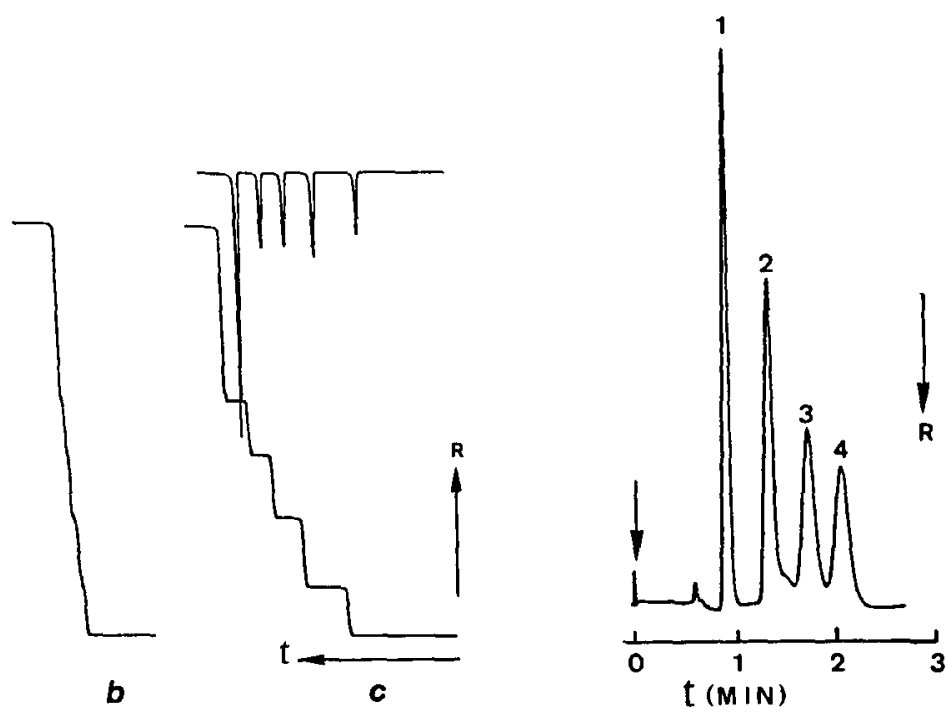

Fig. 3a. Isotachopherogram for the separation of (1) formate, (2) acetate, (3) benzoate and (4) glutamate. The leading electrolyte L was $0.01 M$ hydrochloric acid + and $0.02 M$ histidine at $\mathrm{pH} 6$. The concentrations of the sample ionic species were $5 \cdot 10^{-4} \mathrm{M}$. The terminator $\mathrm{T}$ was $2-(\mathrm{N}$-morpholino)ethanesulphonic acid (MES). The electric current was $25 \mu \mathrm{A}$. (b) As (a) with sample concentrations of $5 \cdot 10^{-5} M$. (c) As (b) with electromigration sampling.

Fig. 4. Electropherogram for the zone electrophoretic separation of (1) formate. (2) acetate. (3) benzoate and (4) glutamate. The concentrations of the sample ionic species were $2.5 \cdot 10^{-5} \mathrm{M}$. The background electrolyte was $0.01 M \mathrm{MES}+0.02 M$ histidine at a pH 6.4. The electric current was $25 \mu \mathrm{A}$. 


\section{DISCUSSION}

Using the new sampling device a known amount of sample ionic species can be introduced and it has advantages over other sampling techniques in which a known sample is introduced. Compared with injection by a syringe this method has the advantage that there is no mixing with other electrolyte solutions, in either the ITP or the CZE mode. Compared with sampling valves the advantage is the absence of rotating parts. Compared with methods applying electromigration it has the advantage of introducing a representative aliquot of the sample (trapping ${ }^{9}$ or splitting $^{5}$ have a similar advantage). To demonstrate the effect of applying electromigration for sampling three ITP experiments were carried out with the equipment described in Fig. 1, from which the isotachopherograms shown in Fig. 3 were obtained. Fig. 3 a shows the separation of formate, acetate, benzoate and glutamate anions in a system of $\mathrm{pH} 6$ and with concentrations of the sample ionic species of $5 \cdot 10^{-4} \mathrm{M}$. The separation was repeated with the same anions at concentrations of $5 \cdot 10^{-5} \mathrm{M}$ (Fig. 3b) and the zone lengths are scarcely visible. In a third experiment (Fig. 3c) the sampling was carried out by electromigration, a dilute sample of $5 \cdot 10^{-5} M$ flowing through the SD for $30 \mathrm{~s}$ with application of an electric current. Although large zone lengths could be obtained, it can clearly be seen that no representative sample aliquot was introduced. The sample introduction follows a moving boundary procedure whereby larger amounts of ionic species with high mobilities are introduced.

With the same sample solution of concentrations $2.5 \cdot 10^{-5} \mathrm{M}$ a zone electrophoretic experiment was also carried out and the electropherogram is shown in Fig. 4. The peaks are clearly visible, showing that very dilute samples easily can be handled using CZE. Further, the SD gives much greater possibilities of automating the sampling procedure both for ITP and CZE.

\section{REFERENCES}

1 F. E. P. Mikkers, F. M. Everaerts and Th. P. E. M. Verheggen, J. Chromatogr., 169 (1979) 11.

2 A. Tsuda, K. Nomura and G. Nakagawa, J. Chromatogr., 264 (1983) 385.

3 J. W. Jorgenson and K. DeArman Lukacs, J. Chromatogr., 218 (198I) 209.

4 J. W. Jorgenson and K. DeArman Lukacs, Science, 222 (1983) 266.

5 M. Deml, F. Foret and P. Bǒcek, J. Chromatogr., 320 (1985) 159.

6 F F. P. Mikkers, F. M. Fveraerts and Th. P. F. M. Verheggen, J. Chromatogr., 169 (1979) 1.

7 F. M. Everaerts J. L. Beckers and Th. P. E. M. Verheggen, Isotachophoresis, Theory, Instrumentation and Applications, Elsevier, Amsterdam. 1976.

8 J. L. Beckers, Th. P. E. M. Verheggen and F. M. Everaerts, 452 (1988) 591.

9 F. E. P. Mikkers, Thesis, Eindhoven University of Technology, 1980, p. 142. 